\title{
Critical crater diameter and asteroid impact seismology
}

\author{
Erik ASPHAUG \\ Earth and Planetary Sciences, University of California, Santa Cruz, California, USA \\ Corresponding author. E-mail: easphaug@ucsc.edu
}

(Received 23 April 2007; revision accepted 07 January 2008)

\begin{abstract}
If impact stress reverberation is the primary gradational process on an asteroid at global scales, then the largest undegraded crater records an asteroid's seismological response. The critical crater diameter $D_{\text {crit }}$ is defined as the smallest crater whose formation disrupts all previous craters globally up to its size; it is solved for by combining relationships for crater growth and for stress attenuation. The computation for $D_{\text {crit }}$ gives a simple explanation for the curious observation that small asteroids have only modest undegraded craters, in comparison to their size, whereas large asteroids have giant undegraded craters. $D_{\text {crit }}$ can even exceed the asteroid diameter, in which case all craters are "local" and the asteroid becomes crowded with giant craters. $D_{\text {crit }}$ is the most recent crater to have formed on a blank slate; when it is equated to the measured diameter of the largest undegraded crater on known asteroids, peak particle velocities are found to attenuate with the 1.2-1.3 power of distance-less attenuative than strong shocks, and more characteristic of powerful seismic disturbances. This is to be expected, since global degradation can result from seismic $\left(\mathrm{cm} \mathrm{s}^{-1}\right)$ particle velocities on small asteroids. Attenuation, as modeled, appears to be higher on asteroids known to be porous, although these are also bodies for which different crater scaling rules might apply.
\end{abstract}

\section{INTRODUCTION}

Asteroids collide with one another, forming craters and disrupting previous topography (Sullivan et al. 2002 and references therein). The most energetic asteroid collisions are catastrophically disruptive (Farinella et al. 1982; Nesvorný et al. 2006), but smaller impacts are more common, producing cratered landscapes (e.g., Chapman 1978). Inbetween are impacts large enough to globally shake the target, disrupting prior landforms (e.g., Richardson et al. 2004; Thomas and Robinson 2005) and resetting the cratering record (Greenberg et al. 1996). The focus here is upon the threshold cratering event that resets all prior surface features up to the scale of the resultant crater. This is called the critical crater diameter $D_{\text {crit }}$ and is represented by the largest undegraded crater on an asteroid.

The planetary bodies that pertain to this study are those whose topography is dominated by impact cratering and impact-induced shaking. This population is likely to include not only typical asteroids, but also small rocky satellites and perhaps some ice-moons and comets. For these impactdominated bodies a correlation can be made between the observed crater population and the body's geomechanical response to impacts. As an example, a highly porous asteroid might be able sustain one or more very large craters without losing its past cratering record, since stress waves are rapidly attenuated in porous media, whereas an asteroid that transmits stress energy efficiently (high $Q$ ) might globally vibrate away its pre-existing topography following a relatively small impact. Formally, if one has data of crater locations, rim radii, degradation states $(x, y, z, r, s)$, a model for interior mechanical characteristics $Q(x, y, z)$, and a corresponding model for stress wave propagation in the asteroid, then in principle an inversion can be performed to solve for the interior, since the wave field corresponding to a given crater formation event is recorded in the erasure and degradation of the smaller craters.

A much simpler approach is taken here. If crater formation and degradation are the result of the same impacting population, and if crater scaling is understood, then two pieces of data about an asteroid (its size and the diameter of its largest undegraded crater) can be used to constrain the attenuation of impact energy at global scales. This simple approach to asteroid seismology might allow a basic understanding of the mechanical structure of an asteroid, and its response to energetic events, through the acquisition of moderate-resolution images. A more comprehensive study of the crater statistics might enable detailed mechanical characterization at low cost, through imaging.

These possibilities stem from the curious observation that 
Table 1. Asteroid diameters, densities, and largest distinct craters. Asteroid diameter assumes an equal-mass sphere. This table is based upon published values, especially Table I from Thomas (1999), but excludes the largest craters that are highly degraded. Thus, for example, the largest undegraded crater on Deimos is not the $\sim 10 \mathrm{~km}$ feature from Table I in Thomas (1999), but the next-largest crater, $\sim 2.5 \mathrm{~km}$ in diameter, which retains clear rim topography. Vesta and Amalthea are included for comparison, although their largest craters may prescribe to a different impact physics. Vesta's giant crater is likely a complex structure wider than the original transient cavity, and Amalthea is a target that is either extremely porous or ice-rich. The densities of Deimos, Phobos, Ida, Mathilde, and Vesta are from Thomas (1999); Itokawa and Eros densities are as from Abe et al. (2006) and Yeomans et al. (2000); Amalthea's density is from Anderson et al. (2005). Other densities are estimated on the basis of taxonomy and/or geology. The values in this table are used as the basis for Fig. 2.

\begin{tabular}{llccl}
\hline Asteroid & $\begin{array}{l}\mathrm{D} \\
(\mathrm{km})\end{array}$ & $\begin{array}{l}\rho \\
\left(\mathrm{g} / \mathrm{cm}^{3}\right)\end{array}$ & $\begin{array}{l}D_{L} \\
(\mathrm{~km})\end{array}$ & $\begin{array}{l}\chi_{\text {obs }}= \\
D_{L} / D\end{array}$ \\
\hline Itokawa & 0.33 & 1.9 & $\sim 0.03$ & 0.1 \\
Dactyl & 1.4 & $\sim 2(?)$ & 0.3 & 0.2 \\
1999 JM8 & $\sim 7$ & $\sim 2(?)$ & 1.5 & 0.2 \\
Deimos & 12.4 & $1.8 \pm 0.3$ & 2.5 & 0.20 \\
Gaspra & 12.4 & $2.7(?)$ & 3.0 & 0.24 \\
Eros & 17 & 2.7 & 5.5 & 0.32 \\
Phobos & 22.2 & $1.9 \pm 0.1$ & 9.4 & 0.45 \\
Ida & 31.4 & $2.6 \pm 0.5$ & 14 & 0.44 \\
Mathilde & 53.0 & $1.3 \pm 0.1$ & 33 & 0.62 \\
Amalthea & 167 & $0.86 \pm 0.1$ & 88 & 0.52 \\
Vesta & 530 & $3.5 \pm 0.4$ & 460 & 0.85 \\
\hline
\end{tabular}

small asteroids have only small undegraded craters, relative to their diameter, while large asteroids have very large ones. Table 1 lists the diameter of the largest undegraded crater $D_{L}$ for a number of asteroids (and possible asteroid-like bodies), plus the size ratio $\chi_{\text {obs }}=D_{L} / D$ where $D$ is the asteroid diameter. Figure 1 shows a number of the asteroids imaged to date, including the Martian satellite Phobos, which along with Deimos is probably a captured asteroid. The figure includes the Arecibo radar target 1999 JM8 (Benner et al. 2002), one of several radar-imaged asteroids to show an undisputed crater.

Thomas (1999) studied the largest craters on small satellites and asteroids, and many of the objects shown above are from his Table I. For this study it is necessary to distinguish asteroids from the possibly comet-like bodies whose global-scale gradation may be unrelated to impact. Vesta and Amalthea are included in the analysis, but are outliers if they succumb to endogenic processes at global scales, such as viscous relaxation. It is also important to consider only the undegraded large craters and not, for instance, such relic features as the hemispheric dichotomy of Deimos, or the flat circular relics on Itokawa.
In the past decade, spacecraft have observed additional asteroids up close, and the Arecibo and Goldstone radio observatories have enabled spectacularly detailed observations of small bodies (e.g., Benner et al. 2002; Ostro et al. 2002, 2006). The trend of asteroid cratering is now rather obvious: the largest asteroids have the largest undegraded craters relative to their diameter. This paper makes sense of that trend in order to learn about interior mechanical properties.

\section{THE LARGEST UNDEGRADED CRATER}

The diameter $D_{L}$ of the largest undegraded crater is by definition not ambiguous, and is adopted as the basis for this model. If one debates whether or not it is a crater, then it is not $D_{L}$. On the $\sim 22 \mathrm{~km}$ diameter Martian satellite Phobos it is Stickney $(\sim 9.4 \mathrm{~km})$. On $\sim 53 \mathrm{~km}$ diameter 253 Mathilde it is Karoo $(33 \mathrm{~km})$, although three other craters (Ishikari, Damodar, and Kuznetsk) are also almost $30 \mathrm{~km}$ diameter. (Mathilde's interesting case of giant crater saturation is considered further below.) On the $\sim 330 \mathrm{~m}$ diameter asteroid 25143 Itokawa, visited by the Hayabusa mission (Fujiwara et al. 2006), a few large craters have been identified, but only on the basis of textural remnants such as circular ponded infill. The largest undegraded crater on Itokawa is at most a few tens of meters in diameter.

Miyamoto et al. (2007) report on an impact crater $\sim 30 \mathrm{~m}$ in diameter, adopted here as $D_{L}$; thus $\chi_{\text {obs }}=0.1$ for Itokawa, the smallest value on any imaged asteroid. According to gravity scaling (discussed below), this crater is formed by an impactor only $\sim 4 \mathrm{~cm}$ in diameter traveling at $5 \mathrm{~km} \mathrm{~s}^{-1}$. This might seem surprising, but turns out to be consistent with the model developed below; nonetheless we need to pay attention to the uncertainties in crater scaling on small asteroids.

A central assumption is that asteroid topographic gradation at global scales is primarily the result of stress waves radiating from large impacts. This ignores other potentially important processes of crater degradation such as ejecta mantling and erosion by small-scale "gardening" impacts, or dust transport by electrical and solar wind effects (see e.g., Robinson et al. 2002) - process which may in fact dominate at smaller scales, but are not expected to control an asteroid's global topography. While in principle any process in which impacts erase prior impacts can be folded into a model for critical crater diameter, only impact stress reverberation is considered here, in light of the following studies of crater retention which indicate that this is the primary gradational process for large-scale craters.

\section{SEISMIC RESURFACING}

The resurfacing of asteroids through impact-induced shaking has been explored using impact hydrodynamics codes (Asphaug and Melosh 1993; Nolan et al. 2001) and linked hydrocode-seismic models (Richardson et al. 2004). 
These and other studies agree that the formation of a sizable crater has the potential to globally erase pre-existing topography on a body tens of kilometers in diameter. Richardson et al. (2004) show that if an asteroid the size of Eros has the seismic properties measured for lunar regolith, then its $\sim 100 \mathrm{~m}$ and smaller scale craters will have disappeared due to reverberations by its most recent large crater, in accordance with the crater statistical data from the NEAR-Shoemaker mission.

Thomas and Robinson (2005) find that the retention of pre-existing craters on the irregular-shaped $(33 \times 13 \times 13 \mathrm{~km})$ asteroid 433 Eros increases with radial distance from its most recent large impact structure, Shoemaker Regio $(\sim 7 \mathrm{~km}$ in diameter). Regions opposite Shoemaker Regio, across the short axis of the asteroid, are degraded as much as regions equidistant along the crater's flanks. Moreover, the striking correlation of crater degradation with radial distance from the impact suggests that the initial pulse, rather than more complex reverberation, governs crater erasure.

These observations justify the simplifying assumption, that in order to degrade a topographic structure of a certain spatial scale $x$, the structure must be shaken by impact with a particle velocity $v \sim \sqrt{g x}$, where $g$ is the average surface gravity of the asteroid, and where $v$ is the peak particle velocity in the initial pulse radiating from the impact. This simple approach to seismic shaking is now combined with scaling relations between crater diameter and projectile radius (Housen et al. 1983; Holsapple 1993) to address the question of why small asteroids have no large craters, relative to their diameter, while large asteroids have huge ones.

\section{CRATER ERASURE ON ITOKAWA}

The smallest asteroid so far visited, $\sim 330 \mathrm{~m}$ in diameter Itokawa, makes a useful initial focus. (Asteroids are assumed spherical in this analysis, when in fact Itokawa and many other asteroids have 2:1 or greater aspect ratios.) Itokawa has the smallest undegraded craters of any asteroid yet imaged $\left(\chi_{\mathrm{obs}} \sim 0.1\right)$, yet is highly fragmented, indicating a history of disruptive collisions. Fujiwara et al. (2006) argue that these rubble-forming collisions occurred when Itokawa was part of a much larger parent body.

Most of Itokawa's mass appears to be $\sim 1 \mathrm{~cm}$ to $\sim 10 \mathrm{~m}$ rubble, although below the visible surface the structure is inferred on the basis of its low bulk density $\left(\sim 1.9 \mathrm{~g} / \mathrm{cm}^{3}\right)$ derived from spacecraft navigation (Abe et al. 2006). Compared to the mass density of ordinary chondrites $\left(\sim 3.4 \mathrm{~g} / \mathrm{cm}^{3}\right)$, which are the closest meteorite analog (Okada et al. 2006), this indicates a porosity as high as $40-50 \%$. Rubble attenuates strong stress pulses (Love et al. 1993) and thus any impactor capable of resetting Itokawa's crater population must be large enough to cause global shaking despite the attenuation. And so, the absence of a large undegraded crater on Itokawa is a puzzle.
If crater formation and degradation on Itokawa are in approximate equilibrium, then there are two possibilities. If the crater that would have formed by the most recent globalresurfacing event was larger than $\sim 30 \mathrm{~m}$, then the event must have finished by erasing its own crater, since there is no larger undegraded crater. This requires that impact reverberations persist for longer than the crater formation time. The formation of a crater that is a significant fraction of the target diameter, in the gravity regime (probably appropriate for cohesionless rubble even on a small asteroid; see below), takes place on a time scale proportional to the free-fall time scale $\sim 1 / \sqrt{G \rho}$, about an hour. If Itokawa has an acoustic velocity of $\sim 200 \mathrm{~m} \mathrm{~s}^{-1}$ (typical for lunar regolith; Kovach and Watkins 1972) then its stress wave crossing time is $\sim 1 \mathrm{~s}$. In order for a crater of diameter $D_{c}$ to be erased by its own impact seismicity, reverberations of particle velocity $\sim \sqrt{g D_{c}}$ must persist for $\sim 10^{3}$ wave crossings, otherwise the crater finishes forming on a quiet asteroid and is preserved. It is doubtful that rubble pile asteroids can reverberate with such high efficiency, although attenuation in rubble may in fact be low for particle velocities comparable to Itokawa's escape velocity, a few $\mathrm{cm} \mathrm{s}^{-1}$.

An alternative is that Itokawa's $\sim 30 \mathrm{~m}$ crater is a "large" structure whose formation erased all previous craters of that size or smaller. That a $30 \mathrm{~m}$ crater can be a global-changing event on Itokawa is consistent with the trends in Table 1; at $\sim 1 / 10$ the asteroid diameter this is not a "small" crater. To have global consequences, it need only trigger global particle velocities of order $\sim 1 \mathrm{~cm} \mathrm{~s}^{-1}$. But this is not a large event compared to traditional cratering studies, and it pushes the limits of what we know about impact cratering and regolith mechanics. How small of an impact can cause global vibrations of the required amplitude? What is the smallest impact that can reset any asteroid's surface?

\section{CRITICAL CRATER DIAMETER}

The critical crater diameter $D_{\text {crit }}$ represents an equilibrium between crater formation and crater erasure. It is the smallest crater whose formation leads to the erasure of all pre-existing craters of its size, making it the most frequent event to result in the appearance of a solitary large crater on an asteroid. $D_{\text {crit }}$ is evaluated by equating the peak particle velocity attained at the farthest reaches of an asteroid during formation of a crater of diameter $D_{c}$, to the shaking velocity required to shake all craters of size $D_{c}$ or smaller beyond recognition. Craters smaller will form faster than they degrade and thus be common, while craters larger will degrade faster than they form, and thus not be present undegraded. Most commonly then $D_{\text {crit }}$ can be equated to the largest undegraded crater $D_{L}$.

Loose surface material in ballistic flight at an angle of $45^{\circ}$ at a velocity $\sqrt{g x}$ will land a distance $x$ away, neglecting asteroid curvature. Surface gravity is $g=2 / 3 \pi \mathrm{G} \rho D$ for a 
spherical homogeneous asteroid of diameter $D$, where $\rho$ the bulk density. To degrade a crater beyond easy recognition, its rim materials must be thrown a distance approximately one crater radius, $D_{c} / 2$. To cause the global degradation of all craters of diameter $D_{c}$ and smaller, the peak particle velocity at all points out to a distance $D$ from the impact must therefore exceed $\sim \sqrt{\left(g D_{c}\right) / 2}$.

Gradational ground motion is modeled as a single pulse whose particle velocity attenuates as a power of the radial distance $r$ from a source. In the far-field elastic limit under radial divergence, particle velocity attenuates as $v_{p} \propto r^{-1}$, conserving energy. Dissipation and dispersion through friction and stress hysteresis lead to an exponent greater than 1 . The attenuation of stress waves of seismic amplitude is not commonly described by a power law; it is more usual to apply a frequency-dependent damping factor $e^{-\omega r / Q}$ multiplicatively to the geometrical wave spreading function $(1 / r$ for radiation from a point source), where $\omega$ is the frequency of the seismic disturbance and $Q$ is the quality factor, a rock property which might itself be amplitude- and frequency-dependent (Knopoff and MacDonald 1958). For the present purpose, we look at power-law attenuation $v_{p} \propto r^{-\alpha}$ because it introduces one unknown variable $(\alpha)$ instead of two $(\omega$ and $Q$ ). Recognizing that $\omega$ may not be independent, perhaps scaling with $\sim c / r_{i}$ where $r_{i}$ is the impactor radius and $c$ the sound speed, one might in principle solve for $Q$ using a similar approach.

Powerful shocks radiating from explosions in geologic media are observed to attenuate as $v_{p} \propto r^{-2}$ (Rodionov et al. 1972; Perret and Bass 1975; Cooper and Sauer 1977). In the far field the waves become elastic, and thus a combined fit to the near-field and far-field data requires an exponent $\alpha \approx 2$ at high energy near the source and $\mathrm{a} \rightarrow 1$ in the elastic limit. The data compiled by Cooper and Sauer (1977), if divided into low-particle-velocity data and high-particle-velocity data, can be fit by a segmented power law with an exponent $\sim 2$ at particle velocities greater than $\sim 10 \mathrm{~m} \mathrm{~s}^{-1}$, and $\sim 1.5$ at lower (e.g., seismic) particle velocities, although attenuation data at low velocity is sparse in the explosion literature.

Extrapolating $\alpha \approx 2$ to the very low stresses associated with global-scale asteroid cratering and terrain disruption is incongruous with the findings of this paper, presumably because the phenomenology of an explosion, under the high strain rate conditions studied in terrestrial laboratory conditions and field studies, is an imperfect analogy for asteroid cratering. Because escape velocity is only about $1 \mathrm{~m} \mathrm{~s}^{-1}$ per km of asteroid radius, low-amplitude waves subject to low attenuation can have "earth-shattering" consequences.

Express the peak particle velocity in radial symmetry from a point source by a power law

$$
v_{p}(r)=v_{i}\left(r / r_{i}\right)^{-\alpha}
$$

where attenuation $\alpha=1$ in the elastic (energy conserving) limit. Antipodal focusing is ignored, as this would make crater erasure more efficient but only locally. The target thus everywhere attains particle velocities $v_{i}\left(D / r_{i}\right)^{-\alpha}$ or greater, where $D$ is asteroid diameter, and this sets the threshold for global erasure.

\section{CRATER SCALING}

Scaling rules may be used to relate the diameter $D_{c}$ of a large crater on an asteroid, to an impactor of radius $r_{i}$ of some density $\rho$ colliding at some velocity $v_{i}$. In the gravity regime, where strength effects are negligible, it is found from the analysis of rocky targets that an impactor of radius

$$
r_{i}=0.41 D_{c}^{1.28} g^{0.28} v_{i}^{-0.56}
$$

creates a crater of diameter $D_{c}$ (Schmidt and Housen 1987). This scaling relation assumes normal incidence, and that the target and impactor have the same density $\rho$. It expresses the transient crater diameter; subsequent expansion of the crater through slumping and viscous modification is ignored, as appears to be appropriate in very low gravity (Vesta being the obvious exception in Table 1; Thomas 1999).

For strength-dominated targets, crater scaling in competent rock is observed to obey a linear relationship between impactor size and target size,

$$
r_{i}=D_{c}\left(Y / \rho v_{i}^{2}\right)^{0.28}
$$

where $Y$ is the strength (times a coefficient of order unity). Because the strength of asteroids is rate- and size-dependent (Housen and Holsapple 1999; Asphaug 2004), and gravity varies widely, it is debated which if either of these scaling rules applies to small asteroids. With $g$ being a millionth that of Earth, small ( $\sim 100 \mathrm{~m})$ asteroids might experience cratering in a fundamentally different mechanical environment than we are used to.

On the basis of numerical simulations of the largest craters on asteroids, it has been argued (Asphaug and Melosh 1993; Asphaug et al. 1996; Nolan et al. 2001; Asphaug et al. 2002) that gravity scaling should predominate for the largest craters even on competent, rocky asteroids, and certainly for rubble piles. For example, the crater diameters produced in hydrocode simulations by Asphaug and Melosh (1993) for crater Stickney forming in an initially competent, rocky Phobos agree with the gravity scaling prediction of Equation 2, and disagree with Equation 3. Smaller craters forming in unconsolidated regolith are also believed to form in the gravity regime (see e.g., Asphaug et al. 1996 and Nolan et al. 2001), although here the effects of internal friction in low-gravity environments is likely to become important (e.g., Holsapple 2004). It may also be that compaction cratering applies (Housen and Holsapple 2003), with its own scaling physics, which would lead to a revised computation for $D_{\text {crit }}$. 
There have been a couple of novel microgravity studies at small scales in cohesionless targets (Colwell et al. 2003; Takagi et al. 2007), and an in situ cratering experiment was conducted on a small body by the Deep Impact mission to comet Tempel 1 (A'Hearn et al. 2005). Because of the voluminous dust production, Deep Impact was unable to determine the final crater diameter, although the dust excavation has been modeled (Richardson et al. 2005) to derive some fundamental aspects of the event. Our understanding of the Deep Impact cratering event and the mass of the comet it struck remain model dependent.

Accepting the above caveats we proceed using Equations 2 and 3 , which can be expressed more generally as

$$
r_{i}=k_{\mathrm{o}} D^{a} D_{c}^{b} v_{i}^{c}
$$

where $a, b$, and $c$ are scaling exponents with $a$ expressing the influence of gravity ( $g$ scales linearly with $D$ for constant density) and $b$ and $c$ expressing the mechanical aspects of rock excavation by impact. If we fix the impact velocity $v_{i}$, target strength $Y$, and target and impactor density $\rho$, then

$$
r_{i}=k_{1} D^{a} D_{c}^{b}
$$

where in the gravity regime

$$
k_{1}^{\text {grav }}=\frac{1}{2}\left(G \rho / v_{i}^{2}\right)^{0.28}, a=0.28, b=1.28
$$

and in the strength regime

$$
k_{1}^{\mathrm{str}}=\left(Y / \rho v_{i}^{2}\right)^{0.28}, a=0, b=1
$$

The above apply to scaling for rocky targets. Gravity regime rules for sand targets (as opposed to rocky targets) do not appreciably alter the results presented below, although as shall be seen gravity scaling and strength scaling lead to different results for small asteroids. Lest the discussion get too broad it is appropriate to fix $v_{i}=5 \mathrm{~km} \mathrm{~s}^{-1}, Y=10^{5} \mathrm{dyn} / \mathrm{cm}^{2}$ (a value typically used for cratering in lunar regolith), and for the general case $\rho=2 \mathrm{~g} / \mathrm{cm}^{2}$. In this case $k_{l}^{\text {str }}=0.013$ and $k_{l}^{\text {str }}=3.8 \cdot 10^{-6}$, both in cgs units. Asteroid density and strength, impact velocity, and the velocity scaling exponent are contained in $k_{1}$, so that the relations to target size and crater diameter are isolated in Equation 5.

Define the normalized critical crater diameter

$$
\chi=D_{\text {crit }} / D
$$

so that e.g., $\chi=0.44$ describes $9.4 \mathrm{~km}$ diameter Stickney on $22.2 \mathrm{~km}$ diameter Phobos. It follows then from either scaling rule that $\chi$ goes as $D$ to some positive power, for all reasonable values of stress wave attenuation. This is shown by solving for $\chi$. A crater of diameter $D_{\text {crit }}$ degrades all prior craters of that size, and thus must attain a peak particle velocity $v_{p}$ at the maximal distance $D$ exceeding the velocity required to shake apart rims of craters $D_{\text {crit }}$ and smaller. Thus $v_{p}^{2}>g\left(D_{\text {crit }} / 2\right)$ or

$$
v_{p}(r)=v_{i} r_{i}^{\alpha} D^{-\alpha}>\left(\frac{2}{3} \pi G \rho D \cdot D_{\text {crit }} / 2\right)^{1 / 2}
$$

Substituting Equations 5 and 8 gives the normalized critical crater diameter

$$
\chi=\left[\left(k_{2} / k_{1}^{\alpha}\right)^{\alpha(1-a-b)+1}\right]^{1 /(b \alpha-1 / 2)}
$$

where $k_{2}=v_{i}^{-1}(\pi G \rho / 3)^{1 / 2}$. For the values used above, in cgs units $k_{2}=7.48 \cdot 10^{-10}$. For strength regime coefficients

$$
\chi \propto D^{1 /(\alpha-1 / 2)}
$$

while for gravity regime coefficients

$$
\chi \propto D^{(1-0.5 \alpha) /(1.28 \alpha-1 / 2)}
$$

For $\alpha>1 / 2$ (all physical values), the exponent is positive in the strength regime. In the gravity regime the exponent is positive if $\alpha<1.8$ - that is, less attenuative than strong shocks in geologic media. The crater scaling and velocity attenuation relations therefore provide a straightforward explanation for the trend in Table 1, that small asteroids lack large craters. Equation 12 can be solved for $\alpha$, making it possible to use a measurement of an asteroid's size and of its largest distinct crater to obtain interior structural information - assuming that it is in production/disruption equilibrium at the scale of global cratering, and that the scaling regime for crater production is known:

$$
\alpha=\left[\ln k_{2}+\ln D+\frac{1}{2} \ln \chi\right]\left[\left[b \ln \chi-(1-a-b) \ln D+\ln k_{1}\right]\right.
$$

\section{ARE ASTEROIDS IN EQUILIBRIUM?}

The model places two requirements upon the impacting population: that craters of diameter $D_{\text {crit }}$ have had time to form, and that large impacts are less common than small ones. Two of the asteroids in the analysis, Itokawa and Mathilde, can be examined in this regard. Mathilde is an ancient asteroid (Davis et al. 1999) surviving in the Main Belt, while Itokawa is a near-Earth asteroid with a dynamical lifetime of perhaps $10 \mathrm{Ma}$. Does Mathilde have more large craters simply because it has been around for longer? Alternatively, because small asteroids are reset globally much more easily than large ones, is Itokawa's cratering record more mature in this context of critical crater equilibrium?

Assume Mathilde's cratering age is $\sim 2$ billion years (Davis et al. 1999) and that its critical crater diameter is somewhat larger than Karoo (as discussed further below). If a $30 \mathrm{~m}$ crater represents $D_{\text {crit }}$ for Itokawa and if $D_{\text {crit }}>33 \mathrm{~km}$ for Mathilde, and if the relative frequency of impact cratering 
events goes inversely with the $\sim 2$ nd power of impactor size $r_{i}$ times the asteroid cross section, then according to gravity scaling (Equation 3) these relative event frequencies are $>3$ $\times 10^{4}$, which means that Itokawa would be just as evolved as Mathilde, in the context of critical crater diameter, if subject to the same impacting population for $\sim 10^{5}$ years. Current studies of the near-Earth population show a cumulative $N_{>D}$ going as $D^{-1.75}$ for $D>200 \mathrm{~m}$, and $D^{-2.75}$ for $D<200 \mathrm{~m}$ (Brown et al. 2005), and the small-scale flux in the Main Belt is not well constrained, so this comparison from near-Earth to Main Belt small impactor populations must be taken as approximate. But it certainly lends support to the assumption that Itokawa's largest craters are in equilibrium.

\section{MODEL RESULTS}

Figure 2 plots results for the asteroids and other small bodies in Table 1. Figure 2a plots the modeled attenuation coefficient $\alpha$ as a function of critical crater diameter, in the gravity regime. Impact velocity $v_{i}$ is fixed as $5 \mathrm{~km} \mathrm{~s}^{-1}$. Figure $2 \mathrm{~b}$ plots $\alpha$ for strength regime scaling, with strength fixed at $Y=10^{5} \mathrm{dyn} / \mathrm{cm}^{2}$. The general trend is that larger asteroids have larger normalized critical crater diameters $\chi$, for a given $\alpha$. The circles plotted along each asteroid curve represent the largest distinct crater that is observed, from Table 1. For all asteroids, if this largest observed crater is near the critical crater diameter, then the deduced attenuation exponent $\alpha$ is surprisingly constant, 1.2 to 1.3 . This includes many different size scales and asteroid geometries - none of these are truly spheres, and the taxonomic types are diverse. If one were to fix this constant at $\alpha=5 / 4$, one could predict nearly all of the largest crater diameters on these small bodies with reasonable accuracy, although as shall be seen this is not a recommendation to do so.

The critical crater diameter is the scale at which cratering "goes global" and results in a solitary distinct crater. All smaller craters-however large $D_{\text {crit }}$ might be - are local events. Mathilde is thus a particularly interesting case, since it is the largest asteroid imaged at sufficient resolution to show that its topography is clearly exogenic, governed by impacts. Mathilde has several craters rivaling Karoo in size; according to gravity-regime scaling it suffered $\sim 5$ impacts by objects, ranging from $\sim 0.8$ to $1.2 \mathrm{~km}$ diameter, without being struck once by an object large enough to disrupt it. This seems odd, and Cheng and Barnouin-Jha (1999) find Mathilde's survival unlikely, and appeal to oblique impacts. But it may be an effect of preservation in the case where $D_{\text {crit }}$ is so large that even hemisphere-spanning craters are, by definition, "local." If, for example, Mathilde's attenuation is somewhat higher than usual, say $\alpha=1.4$, then based on Fig. 2 none of its craters exceeds the critical crater diameter. This would explain the crowding of giant craters, since none of these would result in global degradation. Indeed if Karoo, at $33 \mathrm{~km}$ diameter, represents $D_{\text {crit }}$ and resurfaced the asteroid, then the 5 or more other giant impacts must have happened subsequently, which is highly unusual given that they are in fact almost just as large. Thus $\alpha=1.33$ from Fig. 2a is likely a lower limit on Mathilde's attenuation.

Amalthea is several times larger than Mathilde, and thus may not be in the impact-dominated gradational regime required for this study. Like Vesta, its topography may degrade at the largest scales by endogenic processes such as viscous relaxation; or like a comet, its large-scale topography may be dominated by outbursts, fissuring, or sublimation. Amalthea has two large craters of about equal size, Pan $(88 \mathrm{~km})$ and Gaea $(\sim 80 \mathrm{~km})$. If Amalthea is impact-dominated, and if Pan is close to the critical crater diameter, then its materials are relatively unattenuative according to Fig. 2a, and more like the rocky asteroids for which $\alpha=1.2$. In that case its composition might be ice-rich and competent (given its bulk density of $0.86 \pm 0.1 \mathrm{~g} / \mathrm{cm}^{3}$; Anderson et al. 2005) instead of highly porous rock. But it is premature to make this claim other than hypothetically, recognizing that the theory of critical crater diameter must be buttressed by in situ seismological observations of small bodies, and possibly developed further to account for not just the single largest undegraded crater, but the sizes of the two or three largest undegraded craters.

\section{COMPARISON TO EXPLOSIONS AND SEISMIC ATTENUATION}

The impact cratering literature grew, in part, out of the study of powerful stress waves emanating from high-energy blast events (see for example Rodionov et al. 1972; Perret and Bass 1975; Cooper and Sauer 1977); the ground motion measurements give particle velocities attenuating with distance reported as $\alpha=1.87 \pm 0.05$ (Perret and Bass 1975), $\alpha=2$ (Cooper and Sauer 1977), or $\alpha \sim 1.6$ to 2.2 (Rodionov et al. 1972). Hydrocode models of asteroid collisions, using shock physics codes, exhibit stress wave attenuation in good agreement with the above values; Asphaug and Melosh (1993) calculated particle velocity decay over distance and found good agreement with published values for granite, halite, and basalt. Evidently the irreversible shock effects and other irreversible effects associated with deformation beyond the elastic limit are captured reasonably well by impact hydrocodes.

But how reliably can these results and models be extrapolated to the low particle velocity waves that dominate large-scale asteroidal cratering? A particle velocity of only $\sim 1 \mathrm{~m} \mathrm{~s}^{-1}$ exceeds the escape velocity of a $\sim 1 \mathrm{~km}$ asteroid, blasting it to smithereens, and this calls us to look at a different data set more appropriate to low-amplitude stress wave attenuation. The literature on earthquakes (e.g., Knopoff and MacDonald 1958) shows attenuation behavior that is overall consistent with the modeling results (e.g., Felzer and Brodsky 2006); in terms of seismic field data a power law fit of $\alpha \sim 1.2$ is a typical value.

Should attenuation be higher in a microgravity rubble 

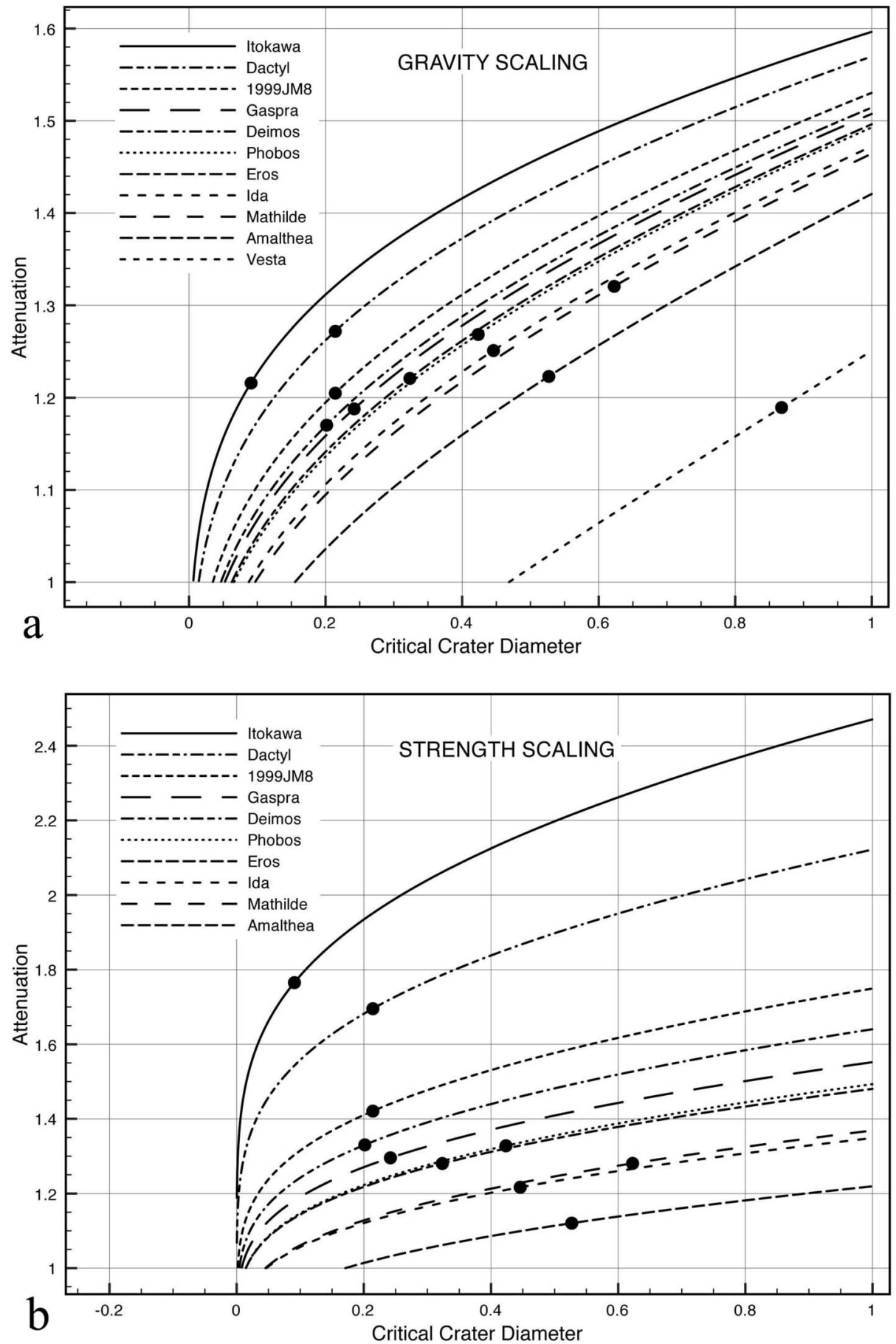

Fig. 2. Plotted below is the attenuation coefficient $\alpha$ as a function of the normalized critical crater diameter $\chi=D_{\text {crit }} / D$ (Equation 13 ), where $D$ is the asteroid diameter. Figure $2 \mathrm{a}$ is a plot for gravity regime scaling, and Fig. $2 \mathrm{~b}$ is for strength regime scaling, for the specific asteroids listed in Table 1. Both plots assume nominal values for impact velocity $\left(5 \mathrm{~km} \mathrm{~s}^{-1}\right)$ and impactor and target density $\left(2 \mathrm{~g} / \mathrm{cm}^{3}\right)$. Figure $2 \mathrm{~b}$ assumes strength properties typical for crater scaling in lunar regolith $\left(Y=10^{5} \mathrm{dyn} / \mathrm{cm}^{2}\right)$. Note the monotonic trend increasing $\chi$ with asteroid diameter, for a given $\alpha$ - the proposed explanation for why larger asteroids have giant craters. Circles on each plot indicate the largest undegraded crater $D_{L}$ observed on each asteroid, from Table 1; if $D_{L} \sim D_{\text {crit }}$ this provides a direct estimate for velocity attenuation $\alpha$ in each asteroid. As noted in the text, for gravity scaling (Fig. 2a), asteroids of low density, or otherwise suspected of being highly porous (e.g., Mathilde, Phobos, Dactyl), appear to have the lowest attenuation coefficients. Deimos has anomalously low attenuation according to the model $(\alpha=1.17)$, perhaps because it is anomalously young following a global resurfacing event and has not attained critical crater equilibrium. For strength scaling (Fig. 2b), $Y=10^{5} \mathrm{dyn} / \mathrm{cm}^{2}$ is adopted as a typical strength of regolith-like materials. Interestingly, for most of the larger asteroids strength scaling gives about the same derived $\alpha$ as gravity scaling. Asteroids as small as Itokawa and Dactyl can only sustain their largest craters in the strength regime if they have high levels of attenuation even at seismic particle velocities. 
pile? There is at present no accepted theory for the broadening and decay of a coherent wave in a granular material (Somfai et al. 2005), and hence there is no a priori guess for $\alpha$, but it is known that distal stress waves in porous media are weak and their rise time is long. Stress waves in rubble with modest granular cohesion could propagate almost elastically once they have attenuated below the threshold of granular cohesion or shear friction. Consider a lunar-like regolith with $Y=$ $10^{5} \mathrm{dyn} / \mathrm{cm}^{2}$ and a sound velocity $c \sim 200 \mathrm{~m} \mathrm{~s}^{-1}$. The peak stress in an elastic stress wave is approximately $\sigma=\rho c v_{p}$. If asteroid Itokawa is made of this material, then it can shake at $\sim 5 \mathrm{~cm} \mathrm{~s}^{-1}$ without exceeding its cohesion, enough to toss surface materials a distance $\sim 30 \mathrm{~m}$, the scale of its largest undegraded crater.

A steeper particle velocity attenuation, appropriate to shocks, has been adopted by the literature on asteroid cratering and catastrophic disruption. In the analytical theory for catastrophic disruption developed by Melosh and Ryan (1997), for instance, the high shock value of $\alpha=2$ is adopted in their criterion that antipodal shaking at $v_{\text {esc }} / 2$ leads to dispersal of the target asteroid, leading to an estimate for $Q^{*}$, the threshold-specific energy per unit mass required for an impact to result in catastrophic disruption. If $\alpha$ is in fact closer to 1.21.3, then their model underestimates (through overdamping) the resultant global particle velocities.

The published hydrocode-based models (Benz and Asphaug 1999) predict $Q^{*}{ }_{D}$ several times greater than Melosh and Ryan (1997). While this and other hydrocodes have been looked at in the purely elastic regime, for which wave propagation is relatively lossless, and in the high-energy regime, where particle velocities decay steeply is agreement with the explosion literature (e.g., Asphaug and Melosh 1993), the study of asteroid catastrophic disruption, involving particle velocities of order a few $\mathrm{cm} \mathrm{s}^{-1}$ to a few $\mathrm{m}$ $\mathrm{s}^{-1}$, warrants a more detailed examination of stress wave behavior.

This aspect of antipodal ground motion is of particular relevance to the concept of diverting a potentially hazardous near-Earth object through the coupling of momentum from an artificial impactor (or equivalent explosion). The momentum coupling parameter $\beta$ is the key factor in the feasibility of such concepts (Belton et al. 2004 and articles therein), which is the ratio of the change in asteroid momentum to the contributed impactor momentum. Because material is thrown back at escaping velocity out of the impact cavity, $\beta$ is typically greater than 1 in a half-space impact. But if attenuation $\alpha$ is low, and escape velocity is low, then an appreciable amount of material can be mobilized to escape from the far side of the asteroid, opposite the intended direction of momentum transfer. This possibility, together with the scientific questions above, can be studied with a relatively simple but carefully monitored asteroid experiment. For example, the detonation of $10 \mathrm{~kg}$ of high explosive on the surface of a $500 \mathrm{~m}$ diameter asteroid of density $2 \mathrm{~g} / \mathrm{cm}^{3}$ will result in antipodal velocity (neglecting focusing) of $\sim 2 \mathrm{~cm} \mathrm{~s}^{-1}$ in the case $\alpha=1.5$, enough to toss rocks a distance $\sim 10 \mathrm{~m}$. If $\alpha=1.2$ then the antipodal velocity is $\sim 20 \mathrm{~cm} \mathrm{~s}^{-1}$, almost equaling the asteroid's escape velocity, causing the escape of considerable material off the back side, and net momentum loss. These differences are easily measurable by a monitoring spacecraft.

\section{CONCLUSIONS}

Through missions, ground based observations, laboratory studies and models, we are beginning to understand the geophysics of small planetary bodies. Investigations have begun to focus upon the near-Earth asteroids, both for science and for pragmatic reasons - the development of near-Earth space as a resource, and the deflection of potentially hazardous objects (Belton et al. 2004). Detailed studies of interior mechanical properties will require orbiters, penetrators and landers, yet much can perhaps be learned on the cheap through a study of the crater population. By measuring the largest undegraded crater on an asteroid we can estimate how it attenuates stress energy, and apply this information towards questions of science and engineering.

Assuming that crater scaling is reasonably well understood for low-gravity targets, and that a single power law can adequately characterize stress wave attenuation, we can make the following conclusions: 1) For a given value of attenuation, the normalized critical crater diameter $\chi=D_{\text {crit }} / D$ increases with asteroid diameter $D$, providing a simple explanation for why small asteroids have no global craters while large asteroids have huge ones. 2) If gravity scaling applies to global cratering, then stress wave particle velocities attenuate globally with about the $1.2-1.3$ power of distance for most asteroids; attenuation appears to be greater $(\alpha \sim 1.3-1.4)$ for asteroids regarded as highly porous. 3) For Mathilde-sized $(\sim 50 \mathrm{~km})$ asteroids with attenuation $\alpha=1.45$ or higher, $D_{\text {crit }}$ exceeds the diameter of the target; if impact cratering governs their large-scale topography, such bodies become saturated with hemisphere-spanning craters.

Acknowledgments - This effort was sponsored by NASA's Planetary Geology and Geophysics program under the project Small Bodies and Planetary Collisions. I thank Kevin Housen for his insightful review, Daniel Scheeres for valuable discussions, and Peter Thomas for making the science of big craters on small bodies such an interesting topic.

Editorial Handling-Dr. Louise Prockter

\section{REFERENCES}

Abe S., Mukai T., Hirata N., Barnouin-Jha O. S., Cheng A. F., Demura H., Gaskell R. W., Hashimoto T., Hiraoka K., Honda T., Kubota T., Matsuoka M., Mizuno T., Nakamura R., Scheeres D. J., 
and Yoshikawa M. 2006. Mass and local topography measurements of Itokawa by Hayabusa. Science 312:1344-1347.

A'Hearn M. F., Belton M. J. S., Delamere W. A., Kissel J., Klaasen K. P., McFadden L. A., Meech K. J., Melosh H. J., Schultz P. H., Sunshine J. M., Thomas P. C., Veverka J., Yeomans D. K., Baca M. W., Busko I., Crockett C. J., Collins S. M., Desnoyer M., Eberhardy C. A., Ernst C. M., Farnham T. L., Feaga L., Groussin O., Hampton D., Ipatov S. I., Li J.-Y., Lindler D., Lisse C. M., Mastrodemos N., Owen W. M., Jr., Richardson J. E., Wellnitz D. D., and White R. L. 2005. Deep Impact: Excavating comet Tempel 1. Science 310:258-264.

Anderson J. D., Johnson T. V., Schubert G., Asmar S., Jacobson R., Johnston D., Lau E. L., Lewis G., Moore W. B., Taylor A., Thomas P. C., and Weinwurm G. 2005. Amalthea's density is less than that of water. Science 308:1291-1293.

Asphaug E. and H. J. Melosh 1993. The Stickney impact of Phobos: A dynamical model. Icarus 101:144-164.

Asphaug E., Moore J. M. Morrison D. Benz W. Nolan M. C., and Sullivan R. A. 1996. Mechanical and geological effects of impact cratering on Ida. Icarus 120:158-184.

Asphaug E., Ryan E., and Zuber M. 2002. Asteroid interiors. In Asteroids III, edited by Bottke W. F., Cellino A., Paolicchi P., and Binzel R. P. Tucson: The University of Arizona Press. pp. 463-484.

Asphaug E. 2004. Interior structures for asteroids and cometary nuclei. In Mitigation of hazardous comets and asteroids, edited by Belton M. J. S., Morgan T. H., Samarasinha N. H., and Yeomans D. K. Cambridge: Cambridge University Press. 464 p.

Asphaug E. 2007. Asteroid surfaces as expressions of seismic interiors (abstract \#2432). 38th Lunar and Planetary Science Conference. CD-ROM.

Belton M. J. S., Morgan T. H., Samarasinha N. H., and Yeomans D. K., eds. 2004. Mitigation of hazardous comets and asteroids. Cambridge: Cambridge University Press. 464 p.

Benz W. and E. Asphaug 1999. Catastrophic disruptions revisited. Icarus 142:5-20.

Benner L. A. M., Ostro S. J., Nolan M. C., Margot J.-L., Giorgini J. D., Hudson R. S., Jurgens R. F., Slade M. A., Howell E. S., Campbell D. B., and Yeomans D. K. 2002. Radar observations of asteroid 1999 JM8. Meteoritics \& Planetary Science 37:779-792.

Brown P. R. E., Spalding D. O., ReVelle E. Tagliaferri and Worden S. P. 2002. The flux of small near-Earth objects colliding with the Earth. Nature 403:165-166.

Chapman C. R. 1978. Asteroid collisions, craters, regolith, and lifetimes. In Asteroids: An exploration assessment, edited by Morrison D. and Wells W. C. NASA Conference Publication \#2053. pp. 145-160.

Cheng A. F. and Barnouin-Jha O. 1999. Giant craters on Mathilde. Icarus 140:34-48.

Colwell J. E. 2003. Low-velocity impacts into dust: Results from the COLLIDE-2 microgravity experiment. Icarus 164:188-196.

Cooper H. F., Jr. and Sauer F. M. 1977. Crater-related ground motions and implications for crater scaling. In Impact and explosion cratering, edited by Roddy D. J., Pepin R. O., and Merrill R. B. London: Pergamon Press. pp. 1133-1163.

Davis D. R. 1999. The collisional history of asteroid 253 Mathilde. Icarus 140:49-52.

Farinella P., Paolicchi P., and Zappala V. 1982. The asteroids as outcomes of catastrophic collisions. Icarus 52:409-433.

Felzer K. R. and Brodsky E. E. 2006. Decay of aftershock density with distance indicates triggering by dynamic stress. Nature 441:735-738.

Fujiwara A., Kawaguchi J., Yeomans D. K., Abe M., Mukai T., Okada T., Saito J., Yano H., Yoshikawa M., Scheeres D. J., Barnouin-Jha O., Cheng A. F., Demura H., Gaskell R. W.,
Hirata N., Ikeda H., Kominato T., Miyamoto H., Nakamura A. M., Nakamura R., Sasaki S., and Uesugi K. 2006. The rubble pile asteroid Itokawa as observed by Hayabusa. Science 312:1330 1334.

Greenberg R., Bottke W. F., Nolan M., Geissler P., Petit J.-M., Durda D. D., Asphaug E., and Head J. 1996. Collisional and dynamical history of Ida. Icarus 120:106-118.

Holsapple K. A. 1993. The scaling of impact processes in planetary sciences. Annual Review of Earth and Planetary Science 21:333-373.

Holsapple K. A. 2004. Equilibrium figures of spinning bodies with self-gravity. Icarus 172:272-303.

Housen K. R., Schmidt R. M., and Holsapple K. A. 1983. Crater ejecta scaling laws - Fundamental forms based on dimensional analysis. Journal of Geophysical Research 88:2485-2499.

Housen K. R. and Holsapple K. A. 1999. Scale effects in strengthdominated collisions of rocky asteroids. Icarus 142:21-33.

Housen K. R. and Holsapple K. A. 2003. Impact cratering on porous asteroids. Icarus 163:102-119.

Kovach R. L. and Watkins J. S. 1973. The velocity structure of the lunar crust. Earth, Moon and Planets 7:63-75.

Knopoff L. and MacDonald G. J. F. 1958. Attenuation of small amplitude stress waves in solids. Reviews of Modern Physics 30: 1178-1192.

Love S. G., Hörz F., and Brownlee D. E. 1993. Target porosity effects in impact cratering and collisional disruption. Icarus 105:216224.

Melosh H. J. and Ryan E. V. 1997. Note: Asteroids: Shattered but not dispersed. Icarus 129:562-564.

Miyamoto H., Yano H., Scheeres D. J., Abe S., Barnouin-Jha O., Cheng A. F., Demura H., Gaskell R. W., Hirata N., Ishiguro M., Michikami T., Nakamura A. M., Nakamura R., Saito J., Sasaki S. 2007. Migration of regolith on the surface of asteroid Itokawa. Science 316:1011-1013.

Nesvorný D., Enke B. L., Bottke W. F., Durda D. D., Asphaug E., and Richardson D. C. 2006. Karin cluster formation by asteroid impact. Icarus 183:296-311.

Nolan M. C., Asphaug E., Greenberg R., and Melosh H. J. 2001. Impacts on asteroids: Fragmentation, regolith transport and disruption. Icarus 153:1-15.

Okada T., Shirai K., Yamamoto Y., Arai T., Ogawa K., Hosono K., and M. Kato 2006. X-ray fluorescence spectrometry of asteroid Itokawa by Hayabusa. Science 312:1338-1341.

Ostro S. J., Hudson R. S., Benner L. A. M., Giorgini J. D., Magri C., Margot J. L., and Nolan M. C. 2002. Asteroid radar astronomy. In Asteroids III, edited by Bottke W. F., Cellino A., Paolicchi P., and Binzel R. P. Tucson: The University of Arizona Press. pp. 151-168.

Ostro S. J., Margot J.-L., Benner L. A. M., Giorgini J. D., Scheeres D. J., Fahnestock E. G., Broschart S. B., Bellerose J., Nolan M. C., Magri C., Pravec P., Scheirich P., Rose R., Jurgens R. F., De Jong E. M., and Suzuki S. 2006. Radar imaging of binary near-Earth asteroid (66391) 1999 KW4. Science 314:1276-1280.

Perret W. and Bass R. 1975. Free-field ground motion induced by underground explosions. Sandia Report SAND 74-0252.

Richardson J. E., Melosh H. J., and Greenberg R. 2004. Impactinduced seismic activity on asteroid 433 Eros: A surface modification process. Science 306:1526-1529.

Richardson J. E., Melosh H. J., Artemieva N. A., and Pierazzo E. 2005. Impact cratering theory and modeling for the Deep Impact mission: From mission planning to data analysis. Space Science Reviews 117:241-267.

Robinson M. S., Thomas P. C., Veverka J., Murchie S. L., and Wilcox B. B. 2002. The geology of 433 Eros. Meteoritics \& Planetary Science 37:1651-1684. 
Rodionov V. N., Adushkin V. V., Kostyuchenko V. N., Nikolaevskii V. N., Romashov A. N., Sadovskii M. A., and Tsvetkov V. M. 1972. Mechanical effect of an underground explosion. Los Alamos, New Mexico: United States Atomic Energy Commission. UCRL-Trans-10676.

Schmidt R. M. and Housen K. R. 1987. Some recent advances in the scaling of impact and explosion cratering. International Journal of Impact Engineering 5:543-560.

Somfai E., Roux J.-N., Snoeijer J. H., van Hecke M., and van Saarlooset W. 2005. Elastic wave propagation in confined granular systems. Physical Review E 72:021301.

Sullivan R. J., Thomas P. C., Murchie S. L., and Robinson M. S. 2002. Asteroid geology from Galileo and NEAR Shoemaker data. In Asteroids III, edited by Bottke W. F., Cellino A., Paolicchi P., and Binzel R. P. Tucson: The University of Arizona Press. pp. 331-350.
Takagi Y., Hasegawa S., Yano H., Yamamoto S., Sugita S., Teramoto K., Honda C., Kurosawa K., Nakada T., Abe M., and Fujiwaraet A. 2007. Impact cratering experiments in microgravity environment (abstract \#1634). 38th Lunar and Planetary Science Conference. CD-ROM.

Thomas P. C. 1999. Large craters on small objects: Occurrence, morphology, and effects. Icarus 142:89-96.

Thomas P. C. and Robinson M. S. 2005. Seismic resurfacing by a single impact on the asteroid 433 Eros. Nature 436:366369.

Yeomans D., Antreasian P. G., Barriot J.-P., Chesley S. R., Dunham D. W., Farquhar R. W., Giorgini J. D., Helfrich C. E., Konopliv A. S., McAdams J. V., Miller J. K., Owen W. M., Jr., Scheeres D. J., Thomas P. C., Veverka J., and Williams B. G. 2000. Radio science results during the NEAR-Shoemaker spacecraft rendezvous with Eros. Science 289:2085-2088. 